\title{
Anti-atherogenic activity of wild grape (Vitis thunbergii) extract antagonizing smooth muscle cell proliferation and migration promoted by neighboring macrophages
}

\author{
SANG-WOOK KANG ${ }^{1}$, MIN SOO KIM ${ }^{1}$, HYUN-SUNG KIM ${ }^{1}$, YONG-JIN LEE ${ }^{2}$ and YOUNG-HEE KANG ${ }^{1}$ \\ ${ }^{1}$ Department of Food and Nutrition, Hallym University, Chuncheon; ${ }^{2}$ Chuncheon \\ Bioindustry Foundation, Chuncheon, Republic of Korea
}

Received December 28, 2011; Accepted February 14, 2012

DOI: 10.3892/ijmm.2012.931

\begin{abstract}
The proliferation and migration of vascular smooth muscle cells (SMCs) play critical roles in intimal thickening and neointimal hyperplasia in early-phase atherosclerosis. This study tested whether wild grape extract (WGE) suppressed the proliferation and migration of human aortic SMCs induced by neighboring macrophages. Cellular expression of fibrogenic connective tissue growth factor (CTGF) and secretion of collagen IV and matrix metalloproteinase (MMP)-2 were determined in SMCs exposed to THP-1-differentiated macrophage-conditioned media. Proliferation was enhanced in SMCs exposed to macrophage-conditioned media collected during the early stage of differentiation, which was attenuated by treatment with $\geq 10 \mu \mathrm{g} / \mathrm{ml}$ WGE. Increased secretion of CTGF and collagen IV macrophage-conditioned media was suppressed in WGE-supplemented SMCs. TGF- $\beta 1$-promoted production of CTGF and collagen IV was suppressed by blocking TGF- $\beta$ receptors of $\mathrm{R} 1$ and $\mathrm{R} 2$ in SMCs. WGE repressed macrophage-conditioned media-upregulated MMP-2 secretion, indicating that WGE had an ability to encumber plaque rupture within atherosclerotic lesions. In addition, $\geq 1 \mu \mathrm{g} / \mathrm{ml}$ WGE ameliorated the migration of SMCs promoted by neighboring macrophages. These results demonstrate that WGE retarded neointimal hyperplasia and thickening within atherosclerotic plaques largely comprising of macrophages and SMCs. Therefore, WGE may be developed as an antiproliferative and anti-migratory agent targeting SMCs in the proximity of newly differentiated and resident macrophages.
\end{abstract}

\section{Introduction}

Vascular inflammatory responses promote vascular smooth muscle cell (SMC) proliferation, migration and matrix synthesis

Correspondence to: Dr Young-Hee Kang, Department of Food and Nutrition, Hallym University, 1 Ockchon-dong, Chuncheon, Kangwon-do 200-702, Republic of Korea

E-mail: yhkang@hallym.ac.kr

Key words: collagen IV, connective tissue growth factor, macrophages, smooth muscle cell migration, wild grape extract which are the major factors that contribute to narrowing of the vessel lumen (1). Vascular inflammation is mediated by cytokines released by macrophages infiltrated to the subendothelium (2). SMCs themselves secrete cytokines such as interleukin (IL)-6 and IL-8, and express cell adhesion molecules such as vascular cell adhesion molecule-1, which may induce the inflammatory phenotype of SMC and functionally regulate monocyte and macrophage adhesion and other processes during atherosclerosis $(2,3)$. In addition, an intimate interaction of endothelial cells with SMC and changes in matrix composition in the vessel wall drive the inflammatory phenotype of SMC.

Transforming growth factor (TGF)- $\beta$, a major deviser of fibro-proliferative responses to tissue damage, regulates cell proliferation, cell migration, matrix synthesis, calcification and immune responses, all key components of the atherosclerotic process (4). Connective tissue growth factor (CTGF, CCN2) is a secreted matricellular protein and matrix component, the functions of which depend on the interactions with other molecules within the microcellular environment (5). CTGF is one of the candidate factors that are thought to mediate the downstream profibrotic actions of TGF- $\beta$ (6). However, its precise role in vessel interstitial fibrogenesis has not yet been clarified. In addition, fibrogenic stimuli such as thrombin or mechanical stretch can induce CTGF expression (7). CTGF induces proliferation of SMC and accumulation of extracellular matrix (ECM) by inducing collagen (8).

Matrix metalloproteinases (MMP) are proteolytic enzymes with the ability to remodel ECM as well as to modulate cellular mediators such as cell adhesion molecules and growth factor receptors (9). Membrane type 1 metalloproteinase (MT1-MMP) not only exhibits pericellular collagenase activity but also activates pro-MMP-2 and pro-MMP-13 to their active forms, which is essential for tumor invasion and angiogenesis $(10,11)$. MT1-MMP plays important roles in the destabilization of atherosclerotic plaques (12). The accumulation of macrophage-derived foam cells in vulnerable regions of atherosclerotic plaques correlates with increased local release of matrix-degrading MMP and weak fibrous cap tissue. In addition, MMPs regulate SMC migration (13).

Wild grapes may refer to Vitis species, and there are literally thousands of grape varieties found throughout the world. The wild grapes have many uses including wine, jams and jellies 
with appetizing flavor, and natural dye with violet/yellow color. Grape extracts and grape polyphenols possess a diverse array of biological actions and may be beneficial in the prevention of some inflammatory-mediated diseases, including atherosclerosis (14). Grape products contain a large variety of antioxidants including resveratrol, catechin, epicatechin and proanthocyanidins (15). Although not exclusive, antioxidant properties of grape polyphenols appear to be central to their mechanism(s) of actions (16).

Wild grape (Vitis thunbergii var. sinuata Rehder) aerial extract (WGE) showed an inhibitory effect on activity of protein-tyrosine phosphatase-1B inducing insulin receptor dephosphorylation, indicative of anti-diabetic activity of WGE (unpublished data). There is currently an intense attention in developing novel anti-diabetic agents from plants used for alternative medicines. Based on the existing evidence that grape polyphenols and extracts have antioxidant and anti-inflammatory properties, this study further investigated whether WGE prevented atherosclerosis pertaining to enhanced SMC proliferation and migration within atherosclerotic plaques. Wild grapes originated from East Asia countries have been used as wild edible greens or edible mountain fruits, but little is known regarding their use for traditional medicines and therapy. SMC migration and fibrogenic activation within atherosclerotic lesions may be potential targets aimed at preventing atherosclerosis. WGE attenuated production of fibrogenic TGF- $\beta 1$ from macrophages, which manipulated the proliferation and migration of neighboring SMC by influencing the production of CTGF and MMP-2. Accordingly, wild grape may be a novel plant for alternative medicinal uses for diabetes-associated atherosclerosis.

\section{Materials and methods}

Materials.M199, RPMI-1640, human epidermal growth factor, hydrocortisone, phorbol 12-myristate 13-acetate (PMA), and 3-(4,5-dimetylthiazol-yl)-diphenyl tetrazolium bromide (MTT) were obtained from Sigma-Aldrich Chemical (St. Louis, MO, USA), unless specifically mentioned elsewhere. Human aortic SMC, fetal bovine serum (FBS), penicillinstreptomycin and trypsin-EDTA were provided by Lonza Walkersville (Walkersville, MD, USA). THP-1 monocytes were purchased from the American Type Culture Collection (Manassas, VA, USA). Human TGF- $\beta 1$ protein and antibodies of human MT-1 MMP and MMP-2 were purchased from R\&D Systems (Minneapolis, MN, USA). Human PDGF-BB antibody was purchased from Peprotech (Rocky Hill, NJ, USA). Antibodies of human TGF- $\beta 1$, TGF- $\beta$ receptor I kinase (TGF- $\beta-\mathrm{R} 1)$ and TGF- $\beta$ receptor II kinase (TGF- $\beta-\mathrm{R} 2)$ were supplied by Cell Signaling Technology (Beverly, MA, USA); human collagen IV antibody was provided by Santa Cruz Biotechnology, Inc. (Santa Cruz, CA, USA); and CTGF antibody was obtained from Abcam (Cambridge, UK). Horseradish peroxidase-conjugated goat anti-rabbit IgG, donkey anti-goat IgG and goat anti-mouse IgG were obtained from Jackson Immuno-Research Laboratories (West Grove, PA, USA).

Dried aerial parts of wild grapes were purchased from Dae Kwang Herb (Chuncheon, Korea). The voucher specimen (RIC-22) was deposited at the Regional Innovation Center
(Hallym University, Chuncheon, Korea). The dried aerial parts $(1 \mathrm{~kg})$ were refluxed for $2 \mathrm{~h}$ using 100\% methanol (10 liters, 3 times) at $74^{\circ} \mathrm{C}$. WGE was solubilized in dimethyl sulfoxide for culturing with cells and the final culture concentration of dimethyl sulfoxide was $\leq 0.05 \%$.

Culture of human aortic SMC and THP-1 monocytes. Cultures of human aortic SMC were maintained at $37^{\circ} \mathrm{C}$ humidified atmosphere of $5 \% \mathrm{CO}_{2}$ in air. Cells were cultured in SMC growth media containing epidermal growth factor, insulin, fibroblast growth factor, FBS and GA-1000. SMC were plated at $90-95 \%$ confluence in all experiments.

THP-1 monocytes were cultured in RPMI-1640 containing $10 \% \mathrm{FBS}$ and $100 \mathrm{U} / \mathrm{ml}$ penicillin and cultures were maintained at $37^{\circ} \mathrm{C}$ humidified atmosphere of $5 \% \mathrm{CO}_{2}$ in air. THP-1 monocytes were incubated with $50 \mathrm{ng} / \mathrm{ml}$ PMA for up to 7 days in order to differentiate THP-1 cells to macrophages. Culture media were collected to prepare THP-1-differentiated macrophage-conditioned media for the incubation of SMC.

At the end of the incubation of SMC in macrophage-conditioned media, the MTT assay was performed to quantitate SMC viability and toxicity (17). SMCs were incubated in a fresh medium with $1 \mathrm{mg} / \mathrm{ml} \mathrm{MTT}$ for $3 \mathrm{~h}$ at $37^{\circ} \mathrm{C}$. After removing unconverted MTT, the purple formazan product was dissolved in isopropanol through gentle shaking. Absorbance of formazan was measured at $\lambda=570 \mathrm{~nm}$ (Bio-Rad Model 550, microplate reader). Non-toxic concentrations of WGE were $\leq 25 \mu \mathrm{g} / \mathrm{ml}$ in $48 \mathrm{~h}$-serum-free culture conditions.

Western blot analysis. Western blot analysis was performed using whole cell lysates prepared from SMC and culture media of SMC and macrophages. SMC lysates were prepared in $1 \mathrm{M}$ Tris- $\mathrm{HCl}$ (pH 6.8) lysis buffer containing $10 \%$ SDS, $1 \%$ $\beta$-glycerophosphate, $0.1 \mathrm{M} \mathrm{Na}_{3} \mathrm{VO}_{4}, 0.5 \mathrm{M} \mathrm{NaF}$ and protease inhibitor cocktail. Proteins of cell lysates and culture media were fractionated by electrophoresis on $8-15 \%$ SDS-PAGE gels. Nonspecific binding was blocked by soaking the nitrocellulose membrane in TBS-T buffer [0.5 M Tris- $\mathrm{HCl}(\mathrm{pH} 7.5)$, $1.5 \mathrm{M} \mathrm{NaCl}$, and $0.1 \%$ Tween-20] containing 5\% nonfat dry milk for $3 \mathrm{~h}$. The membranes were incubated overnight with primary anti-human PDGF-BB, collagen IV, CTGF, TGF- $\beta 1$, TGF- $\beta$-R1, TGF- $\beta-R 2$, MMP-2 or MT1-MMP. After three washes with TBS-T buffer, the membranes were incubated for $1 \mathrm{~h}$ with goat anti-rabbit $\mathrm{IgG}$, goat anti-mouse $\mathrm{IgG}$, or donkey anti-goat IgG conjugated to horseradish peroxidase (Jackson ImmunoReaserch Laboratories). The protein levels were measured with Supersignal West Pico Chemiluminescence detection reagents (Pierce Biotechnology, Rockford, IL, USA) and Konica X-ray film (Konica, Tokyo, Japan). Incubation with anti-human $\beta$-actin was performed for comparative control.

SMC proliferation. SMC proliferation was assessed using an in vitro scratch assay. SMCs were seeded at 50,000 cells onto a 24-well plate and incubated for $24 \mathrm{~h}$ in a growth media containing $10 \%$ FBS. SMC on the well were scratched away horizontally using a P100 pipette tip. After scratching, injured cells were incubated for another $24 \mathrm{~h}$ in serum-free medium with macrophage-conditioned media with and without $1-25 \mu \mathrm{g} / \mathrm{ml}$ WGE. Images of the scratches were photographed from five random microscopic fields of view along the scratch. 
A reduction in the scratched area indicates a sign of cell migration. Increase of proliferated (migrated) cells was measured by the MTT assay.

Gelatin zymography. For the measurement of MMP-2 activity from culture media, gelatin zymography was performed, as previously described. Briefly, culture media were subjected to electrophoresis on $8 \%$ SDS-PAGE (0.3 M Tris- $\mathrm{HCl}$, $\mathrm{pH} 6.8,4 \%$ SDS, $20 \%$ glycerol and $0.03 \%$ bromophenol blue) co-polymerized with $0.1 \%$ gelatin as the substrate. After completing electrophoresis, gels were incubated for $1 \mathrm{~h}$ at $37^{\circ} \mathrm{C}$ in a $2.5 \%$ Triton X-100 solution, washed in $50 \mathrm{mM}$ Tris- $\mathrm{HCl}$ buffer (pH 7.5) for $30 \mathrm{~min}$, and incubated for $20 \mathrm{~h}$ in $50 \mathrm{mM}$ Tris-HCl buffer (pH 7.5) containing $200 \mathrm{mM} \mathrm{NaCl}, 10 \mathrm{mM}$ $\mathrm{CaCl}_{2}$ and $0.05 \%$ Brij-35. The gels were stained with $0.1 \%$ Coomassie Brilliant Blue G-250, 2\% acetic acid and $45 \%$ methanol, and then destained in a solution with $30 \%$ methanol and $10 \%$ acetic acid.

Cell transmigration assay. The transmigration assay was carried out using a gelatin-coated Transwell apparatus with $8 \mu \mathrm{m}$ pore size (Costar, Corning, NY, USA). The lower sides of the transwell membranes were coated with $10 \mu \mathrm{g} / \mathrm{ml}$ gelatin B and allowed to dry at $25^{\circ} \mathrm{C}$ for $1 \mathrm{~h}$. The transwells were assembled in a 24-well plate, and the lower chambers were filled with the culture media. Cells $(30,000$ cells/well) were added to each upper chamber and the plate was incubated at $37^{\circ} \mathrm{C}$ in $5 \% \mathrm{CO}_{2}$ for $24 \mathrm{~h}$. Cells that had migrated to the lower surface of the filters were stained with toluidine blue, and cells that migrated were counted and photographed in the 2-3 microscopic fields per well using a microscopy with a CCD camera $\left(\right.$ Motic $^{\circledR}$, Wetzlar, Germany).

Data analysis. Values are represented as mean \pm SEM of separate experiments. Statistical analyses were conducted using the Statistical Analysis Systems statistical software package (SAS Institute, Cary, NC, USA). Significance was determined by one-way ANOVA followed by the Duncan multiple range test for multiple comparisons and P-values $<0.05$ were considered statistically significant.

\section{Results}

Inhibition of SMC proliferation and mobility by WGE. Vascular SMC proliferation and migration takes place during the atherosclerotic process. Monocytes/macrophages are known to produce cytokines and other cellular mediators which can regulate gene expression, differentiation and growth of SMC $(18,19)$. This study examined whether newly infiltrated monocytes/macrophages provoked SMC proliferation within atherosclerotic lesions and whether the proliferation was inhibited by supplementing WGE. The addition of THP-1differentiated macrophage-conditioned media collected daily for a 7 day differentiation period substantially promoted SMC growth (Fig. 1A). When cells were incubated in macrophageconditioned media collected on Days 3-4 of differentiation, cell growth increased by $\approx 20-30 \%$. This indicated that cellular mediators secreted in media by macrophages may modulate SMC growth. Consistently, PDGF-BB secretion of macrophages was enhanced in a similar manner to SMC growth

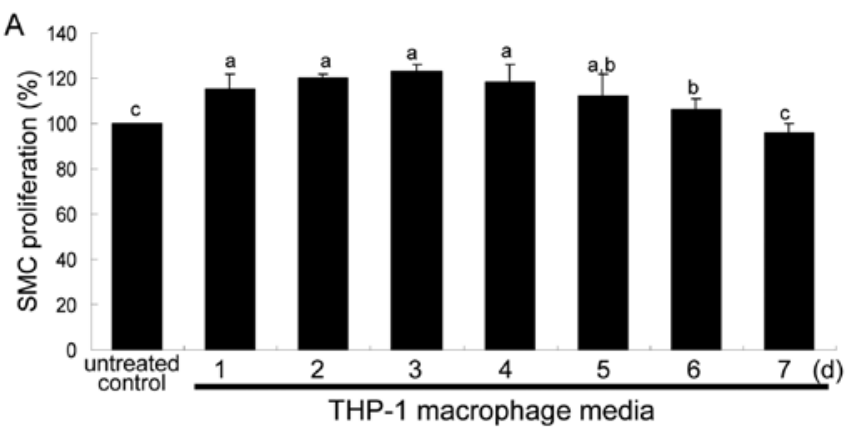

$\mathrm{B}$

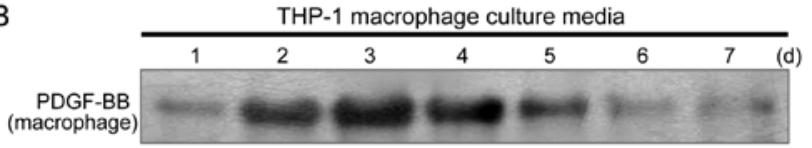

C

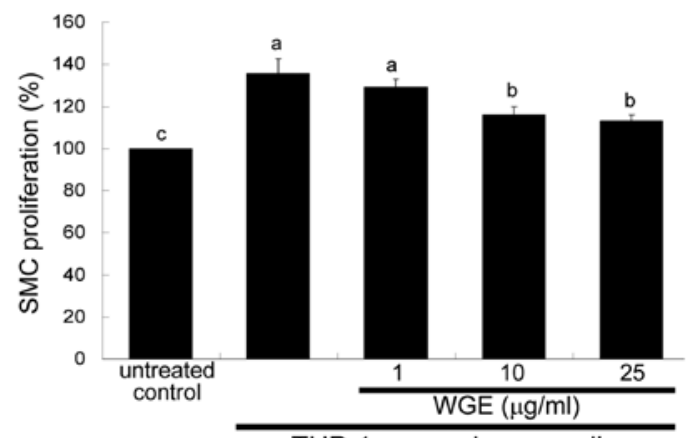

THP-1 macrophage media

Figure 1. Time course responses of (A) SMC growth and toxicity and (B) PDGF secretion in THP-1-differentiated macrophage-conditioned media collected daily for 7 days; (C) inhibitory effects of WGE on growth of SMC treated with macrophage-conditioned media. Values of cell viability (A and C) are mean \pm SEM $(n=5)$ and are expressed as percent cell survival relative to untreated controls (cell viability, 100\%). Values not sharing a common letter are different at $\mathrm{P}<0.05$. PDGF-BB secretion was measured by using western blot analyses with macrophage-conditioned media (B). Representative blots are typical of three independent experiments.

(Fig. 1B). Accordingly, it was assumed that PDGF-BB was one of the mitogenic mediators secreted in the conditioned media. However, the presence of $\geq 10 \mu \mathrm{g} / \mathrm{ml}$ WGE significantly attenuated cell growth (Fig. 1C), indicating that WGE may antagonize mitogenic activity of PDGF-BB.

To investigate the inhibitory effects of WGE on SMC proliferation, the in vitro scratch assay was performed (Fig. 2A). Macrophage-conditioned media containing WGE inhibited the scratch recovery at $24 \mathrm{~h}$ post-injury, suggesting that WGE retarded vascular SMC proliferation was triggered by neighboring macrophages. In addition, WGE attenuated SMC motility $24 \mathrm{~h}$ after the scratch injury (Fig. 2B).

Suppression of collagen IV and CTGF induction by WGE. Acute coronary syndromes often result from rupture of vulnerable plaques. The collagen content of plaques probably regulates their stability (20), and CTGF is responsible for plaque stabilization after stroke (21). This study examined whether macrophages influenced SMC production of collagen IV and CTGF. THP-1-differentiated macrophage-conditioned media collected on Days 3-4 of differentiation increased collagen IV secretion from SMC, which was diminished by WGE in dose-dependent manner (Fig. 3A). The CTGF production 
A

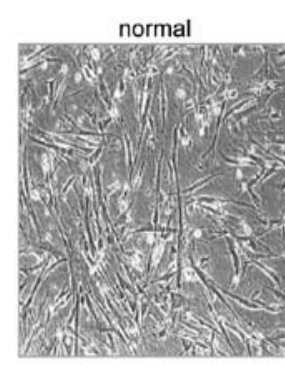

scratched

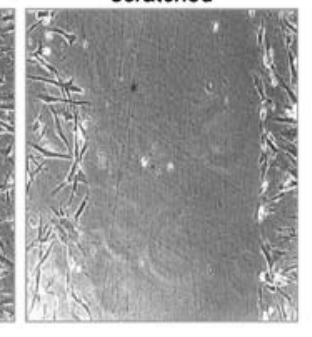

B

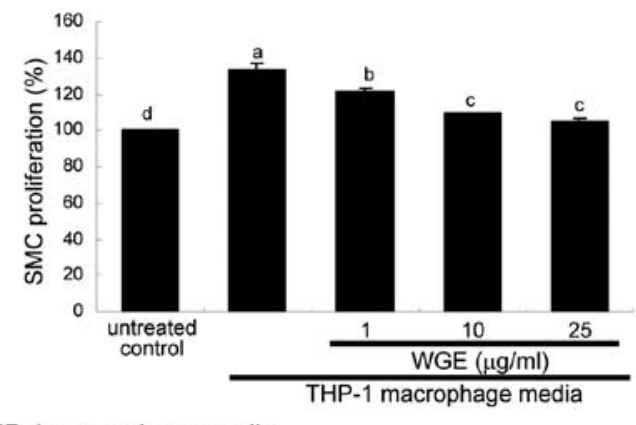

THP-1 macrophage media

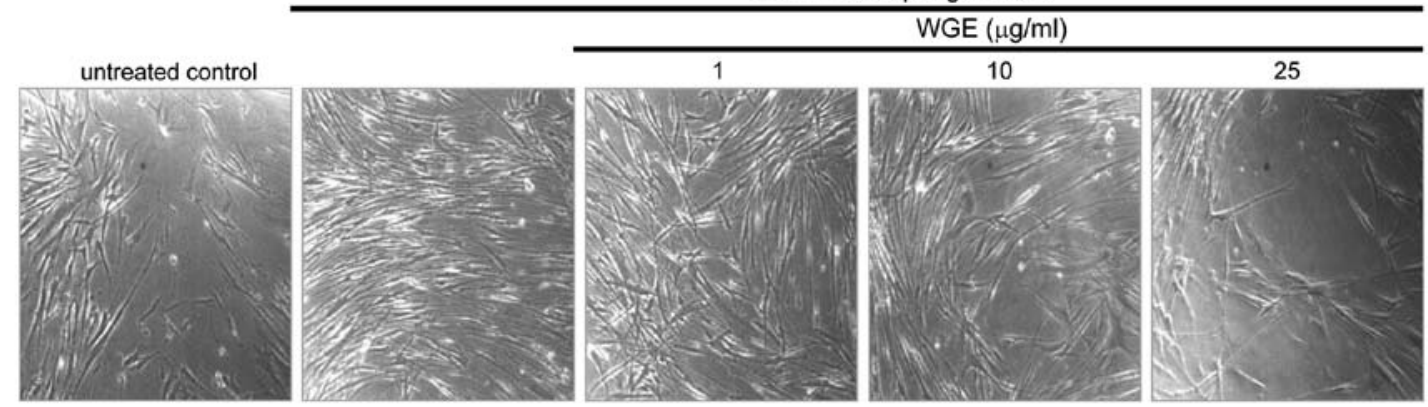

Figure 2. WGE inhibition of proliferation of SMC exposed to macrophage-conditioned media. (A) SMC were scratched away horizontally using a pipette tip. After scratching, cells were incubated for another $48 \mathrm{~h}$ in macrophage-conditioned media containing 1-25 $\mu \mathrm{g} / \mathrm{ml} \mathrm{WGE}$. The scratched area on each well was photographed. (B) After SMC were cultured for $48 \mathrm{~h}$ in macrophage-conditioned media with and without $1-25 \mu \mathrm{g} / \mathrm{ml}$ WGE, the MTT assay was performed. The bar graphs for SMC proliferation represent mean \pm SEM from five independent experiments with multiple estimations. Values are expressed as percent cell survival relative to untreated control cells (cell viability, 100\%). Values not sharing a common letter are different at $\mathrm{P}<0.05$.

A

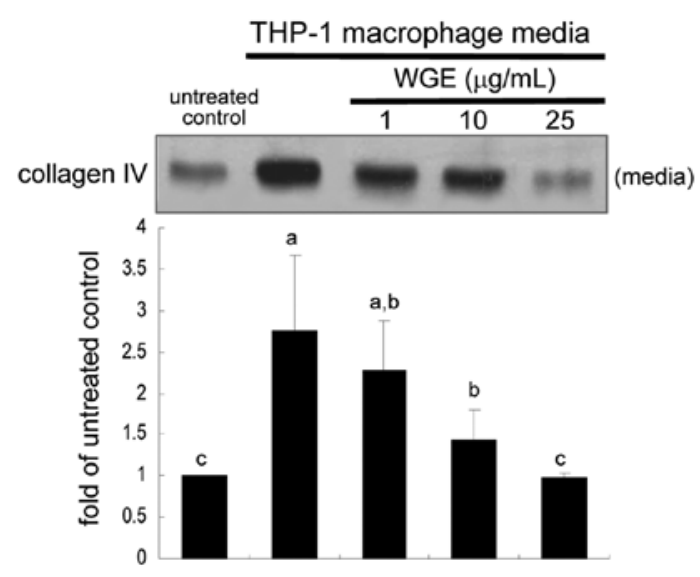

B

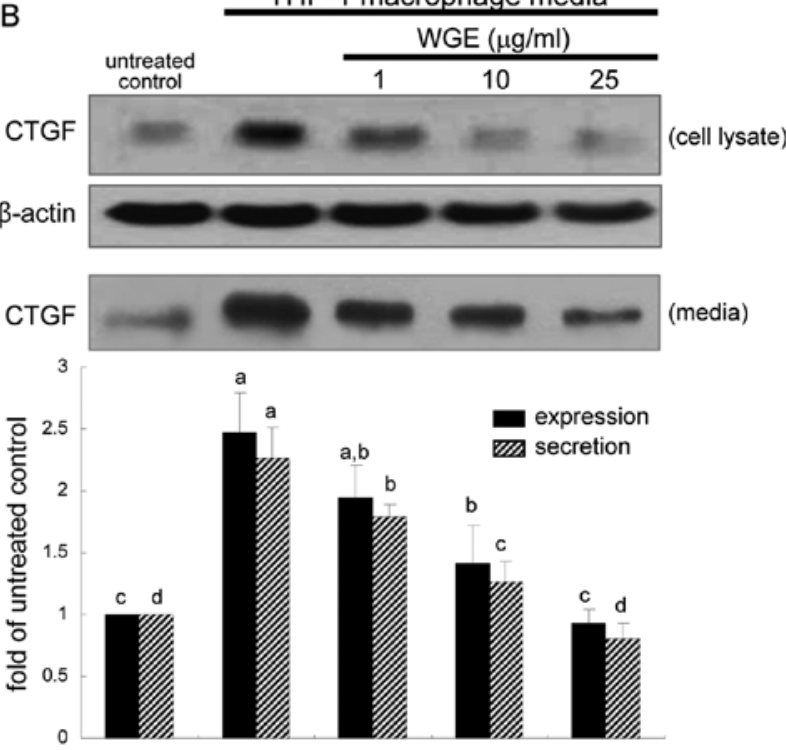

Figure 3. Western blot analysis data showing (A) suppression of collagen IV secretion and (B) CTGF production of SMC by WGE in THP-1-differentiated macrophage-conditioned media. SMC were incubated with and without 1-25 $\mu \mathrm{g} / \mathrm{ml} \mathrm{WGE} \mathrm{in} \mathrm{macrophages} \mathrm{conditioned} \mathrm{media} \mathrm{for} 48 \mathrm{~h}$. Equal amounts of cell lysate proteins and equal volumes of culture media were subject to western blot analysis with a primary antibody against collagen IV or CTGF (3 separate experiments). $\beta$-actin protein was used as an internal control for cellular expression of CTGF. Bar graphs (mean \pm SEM) represent quantitative densitometric results of upper bands. Values not sharing a common letter are significantly different at $\mathrm{P}<0.05$.

was significantly elevated in SMC exposed to macrophage culture media collected in serum-free conditions (Fig. 3B). When 1-20 $\mu \mathrm{g} / \mathrm{ml}$ WGE was applied to SMC, the expression and secretion of CTGF were dose-dependently retarded. The inhibition of collagen IV secretion by WGE was most likely due to reduced CTGF production.
This study further examined whether the production of collagen IV and CTGF from SMC entails TGF- $\beta 1$ activation. The production of collagen IV and CTGF were enhanced in SMC exposed to $10 \mathrm{ng} / \mathrm{ml}$ TGF- $\beta 1$ (Fig. 4). When TGF- $\beta 1$-activated SMC were treated with 1-25 $\mu \mathrm{g} / \mathrm{ml}$ WGE, cellular expression and secretion of CTGF were dose-dependently lessened, 
A

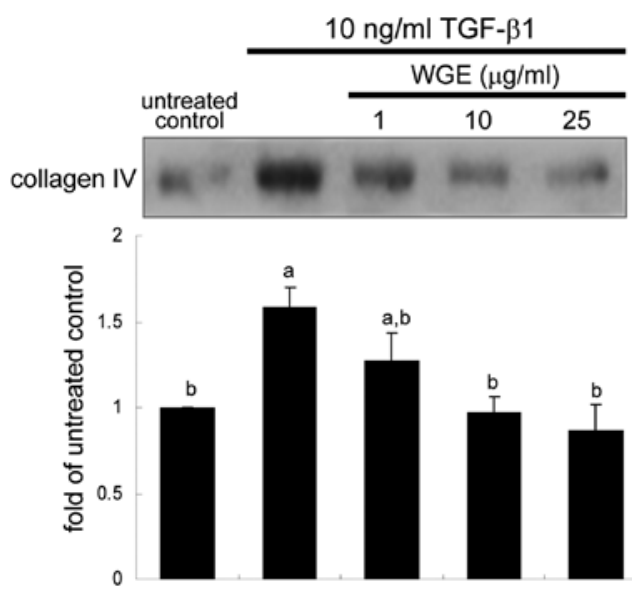

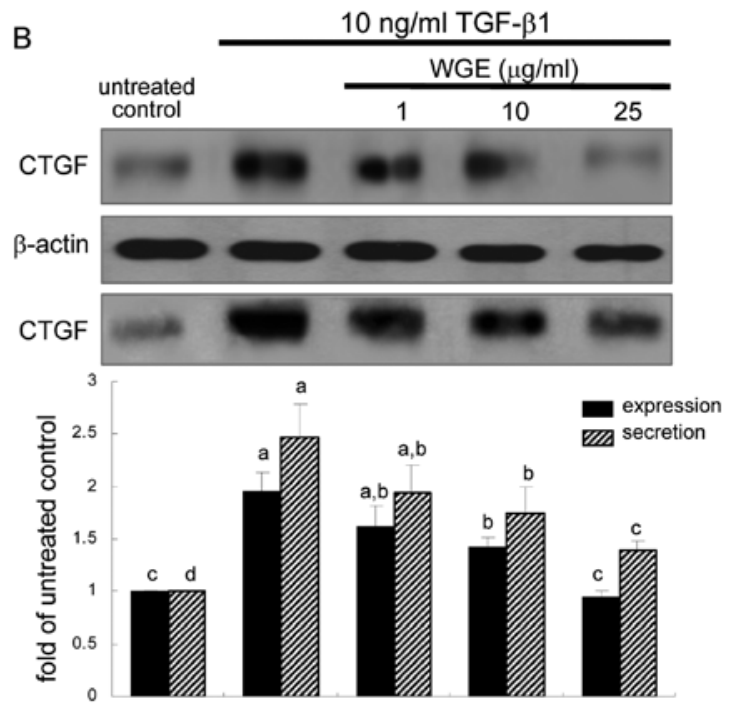

Figure 4. Diminution of TGF- $\beta 1$-induced collagen IV secretion (A) and CTGF production (B) of SMC by WGE. SMC were incubated with 10 ng/ml TGF- $\beta 1$ in the absence and presence of $1-25 \mu \mathrm{g} / \mathrm{ml}$ WGE for $48 \mathrm{~h}$. Equal amounts of cell lysate proteins and equal volumes of culture media were subject to western blot analysis with a primary antibody against collagen IV and CTGF (3 separate experiments). $\beta$-actin protein was used as an internal control for cellular expression of CTGF Bar graphs (mean $\pm \mathrm{SEM}$ ) represent quantitative densitometric results of upper bands. Values not sharing a common letter are significantly different at $\mathrm{P}<0.05$.

A

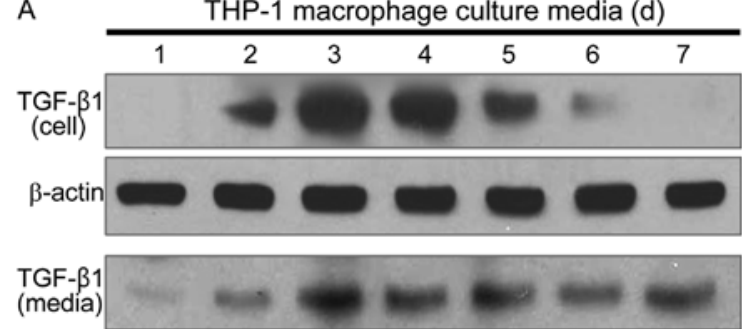

B

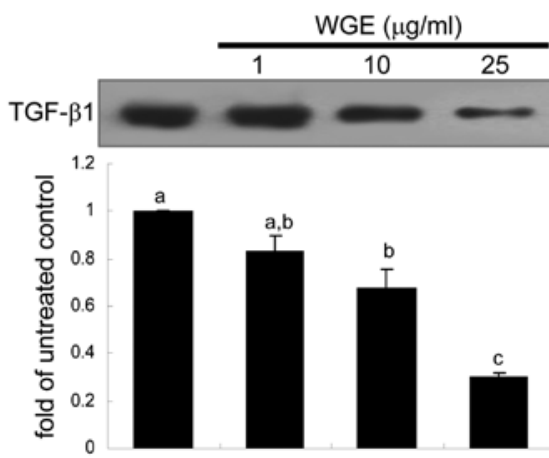

Figure 5. Temporal production of TGF- $\beta 1$ of THP-1-differentiated macrophages (A) and its inhibition by WGE (B). Equal amounts of cell lysate proteins and equal volumes of culture media were subjected to western blot analysis with a primary antibody against TGF- $\beta 1$ ( 3 separate experiments). The bar graphs (mean $\pm \mathrm{SEM}$ ) represent quantitative densitometric results of upper bands. Values not sharing a common letter are significantly different at $\mathrm{P}<0.05$.

being concomitant with a diminution of collagen IV secretion. Accordingly, WGE may be effective in inhibiting secretion of matrix proteins such as collagen IV in the development of the atherosclerotic plaque. Furthermore, TGF- $\beta 1$ appeared to be one of the mediators contained in macrophage-conditioned media.

This study examined whether TGF- $\beta 1$ is notably induced during macrophage differentiation by PMA, influencing the production of matrix proteins from SMC. The cellular expression and secretion of TGF- $\beta 1$ reached a maximum within 3 days after initiating differentiation (Fig. 5A). WGE suppressed the increased TGF- $\beta 1$ secretion in THP-1-differentiated macrophages was by (Fig. 5B).

Blockade of TGF- $\beta$ receptors by WGE. This study investigated the inhibitory effects of WGE on TGF- $\beta$ receptors of SMC. As expected, $10 \mathrm{ng} / \mathrm{ml}$ TGF- $\beta 1$ induced its receptors of R1 and R2 in SMC (Fig. 6A). The induction of TGF- $\beta-\mathrm{R} 1$ and -R2 was attenuated in a dose-dependent manner by $1-25 \mu \mathrm{g} /$ $\mathrm{ml}$ WGE. Similarly, macrophage-conditioned media elevated the expression of TGF- $\beta-\mathrm{R} 1$ and -R2, which was also reversed by $\geq 10 \mu \mathrm{g} / \mathrm{ml} \mathrm{WGE} \mathrm{(Fig.} \mathrm{6B).} \mathrm{It} \mathrm{is} \mathrm{inferred} \mathrm{that} \mathrm{TGF-} \beta 1$ in macrophage-conditioned media was secreted enough to stimulate TGF- $\beta-\mathrm{R} 1$ and $-\mathrm{R} 2$ of SMC.

Western blot analysis was used to address that WGE had a capability to suppress secretion of matrix-degrading proteins from SMC. The MT-1 MMP expression was enhanced in SMC incubated with macrophage-conditioned media for $24 \mathrm{~h}$ (Fig. 7A). Also, the MMP-2 secretion was increased from SMC exposed to macrophage-conditioned media collected for $24 \mathrm{~h}$ in serum-free conditions. The presence of WGE did not alter the cellular levels of MT-1 MMP in SMC neighboring to macrophages. Nevertheless, the MMP-2 secretion was nearly abolished by $\geq 10 \mu \mathrm{g} / \mathrm{ml} \mathrm{WGE} \mathrm{(Fig.} \mathrm{7B).} \mathrm{Consistently,}$ gelatin zymography revealed that $\geq 10 \mu \mathrm{g} / \mathrm{ml}$ WGE abrogated the proteolytic MMP-2 activity of SMC stimulated in macrophage-conditioned media (Fig. 7C).

Retardation of TGF- $\beta 1$-induced transmigration of SMC by $W G E$. An inhibitory effect of WGE on SMC migration was assessed using a transmigration assay. TGF- $\beta 1$ substantially accelerated SMC transmigration, while $\geq 1 \mu \mathrm{g} / \mathrm{ml}$ WGE significantly disturbed the transmigration of TGF- $\beta 1$ stimulated SMC (Fig. 8). Intimal interstitial MMP-2 played an important role in the SMC migration from vascular media to the intima. In addition, it is assumed that MMP-2 is involved in plaque rupture within atherosclerosis lesions. Collagen IV 

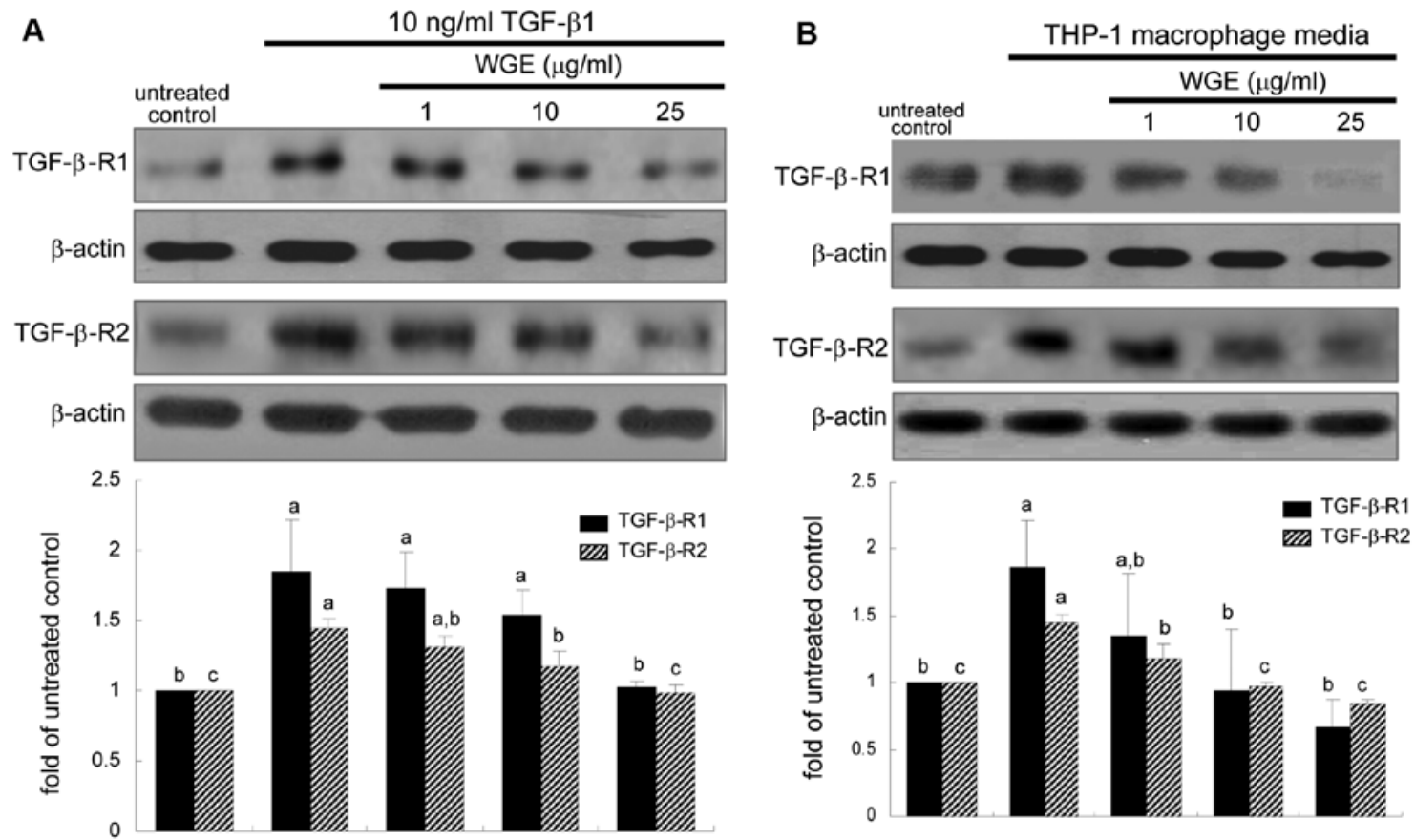

Figure 6. WGE inhibition of TGF- $\beta$-R1 and -R2 induction of SMC by (A) TGF- $\beta 1$ or (B) THP-1-differentiated macrophage-conditioned media. SMC were incubated for $48 \mathrm{~h}$ with $10 \mathrm{ng} / \mathrm{ml}$ TGF- $\beta 1$ or macrophage-conditioned media in the absence and presence of $1-25 \mu \mathrm{g} / \mathrm{ml}$ WGE. Equal amounts of cell lysate proteins were subject to western blot analysis with a primary antibody against TGF- $\beta$-R1 or -R2. $\beta$-actin protein was used as an internal control. The bar graphs (mean $\pm \mathrm{SEM}, \mathrm{n}=3$ ) represent quantitative densitometric results of the bands. Values not sharing a common letter are significantly different at $\mathrm{P}<0.05$.
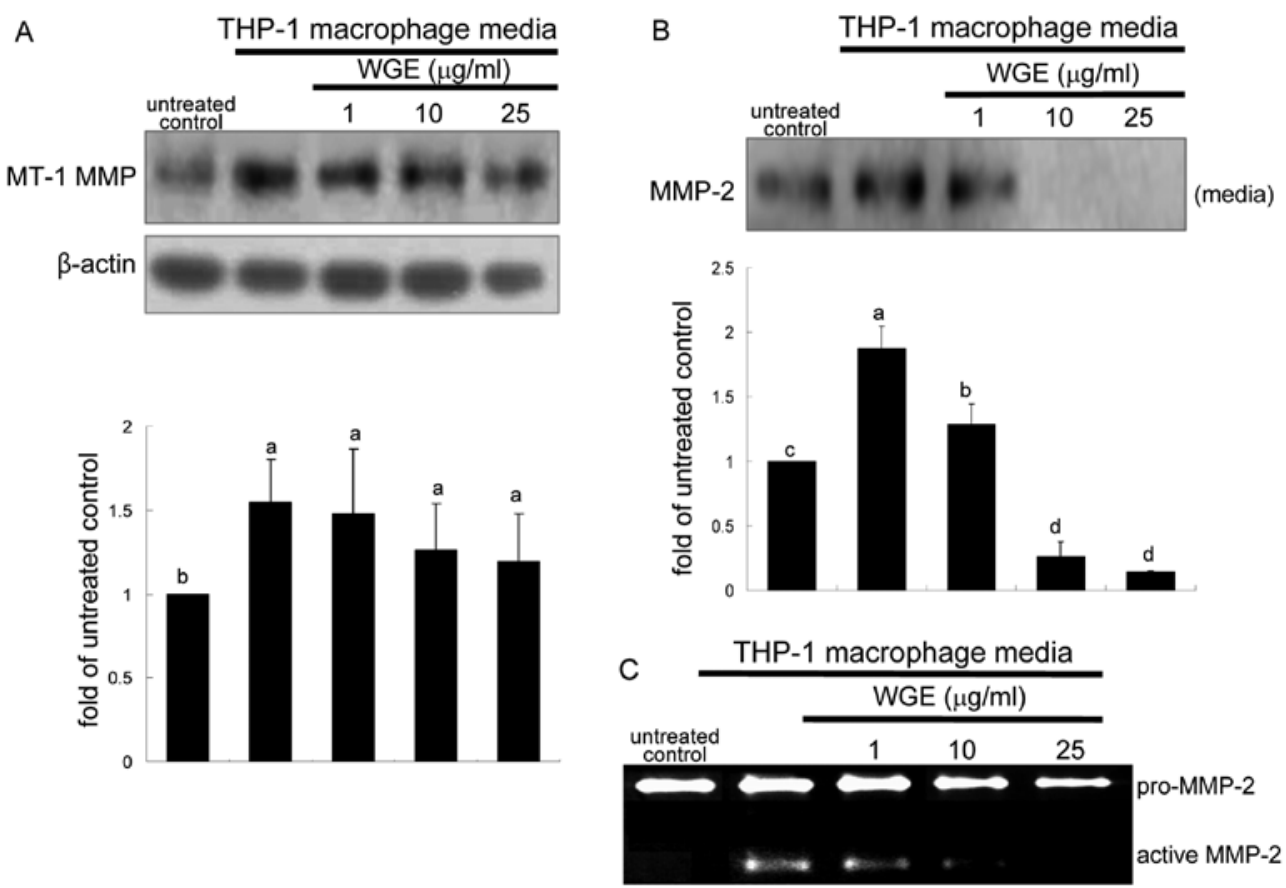

Figure 7. Effects of WGE on (A) MT-1 MMP induction, (B) MMP-2 secretion and (C) activity of SMC in THP-1-differentiated macrophage-conditioned media. SMC were incubated with and without 1-25 $\mu \mathrm{g} / \mathrm{ml}$ WGE in macrophage-conditioned media collected for $36 \mathrm{~h}$. Equal amounts of cell lysate proteins and equal volumes of culture media were subjected to western blot analysis (A and B) with a primary antibody against MT1-MMP and MMP-2 (3 separate experiments). $\beta$-actin protein was used as an internal control. The bar graphs (mean \pm SEM) represent quantitative densitometric results of the western blotting bands. Values not sharing a common letter refer to significant differences at $\mathrm{P}<0.05$. (C) Gelatin zymography was performed for the measurement of the enzyme activity of MMP-2. Gelatinolytic activity was detected as unstained bands against the background of Coomassie blue-stained gelatin.

accumulation by the CTGF and increased matrix-degrading activity of MMP-2 in the intima due to neighboring macrophages appeared to facilitate SMC migration and increase
SMC motility, all of which were antagonized by treatment with WGE. The MMP-2 data (Fig. 7B and C) supported the migration data (Fig. 8). 


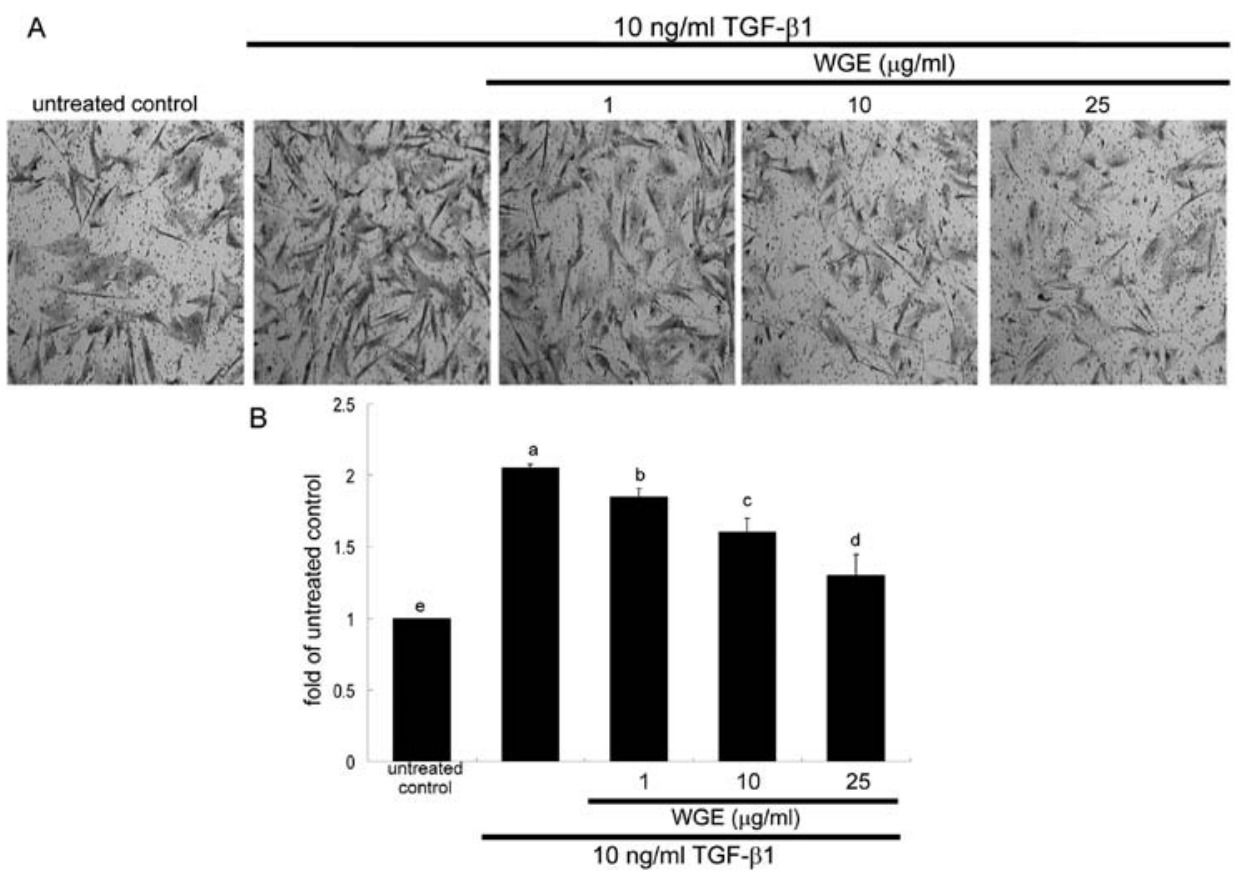

Figure 8. WGE inhibition of transmigration of TGF- $\beta 1$-treated SMC. SMC were cultured on gelatin-coated transwells with $8 \mu \mathrm{m}$ pore-filters. Cells transmigrated for $24 \mathrm{~h}$ onto the lower surface of the filter were stained with toluidine blue and counted. The bar graphs (mean \pm SEM, $\mathrm{n}=4$ ) represent the number of cells transmigrated. Values not sharing a common letter refer to significant differences at $\mathrm{P}<0.05$.

\section{Discussion}

The present study elucidated that cellular mediators, such as cytokines and growth factors released from monocyte-derived macrophages were involved in SMC hyperplasia and intimal thickening. It was explored that WGE suppressed proliferation, ECM production and migration of SMC all responsible for neointimal hyperplasia. SMC were incubated in THP-1differentiated macrophage-conditioned media in order to mimic the microenvironment of SMC disposal in close proximity of newly differentiated and resident macrophages within atherosclerotic plaques. In the advanced stage of atherosclerosis, SMC and migrated monocytes/macrophages are neighbors within intimal compartments.

There are literally thousands of grape varieties found throughout the world. The wild grapes have been used as edibles, for medicinal purposes, and as natural dyes. Grape extracts and polyphenol compounds including resveratrol, catechin, epicatechin, and proanthocyanidins exhibit many biological actions and may be beneficial in preventing some inflammatory-mediated diseases including atherosclerosis (14). Antioxidant properties of grape polyphenols would be central to their mechanism(s) of action $(15,16)$. This study investigated that WGE blocked proliferation and fibrogenic and migratory activity of SMC triggered by newly migrated and resident macrophages in close proximity within atherosclerotic plaques. It means that WGE may be an alternative to ameliorate secretion of macrophage mediators such as PDGF and TGF- $\beta 1$ that may play a bridge role in regulating atherosclerosis by influencing the SMC proliferation and/or migration during plaque and rupture development. Therefore, this study was conducted to evaluate the effects of WGE on the TGF- $\beta 1$ secretion of macrophages to manipulate the proliferation and migration of SMC.
SMC migration from the media to the intima constitutes a critical step in the development of atherosclerotic diseases (22). Modulation of intimal SMC proliferation may be crucial in intimal thickening and vascular reparative responses after angioplasty and vascular injury (23). Vascular inflammatory responses promote proliferation, migration and matrix synthesis of SMC, the major factors contributing to narrowing of the vessel lumen (1). Numerous cytokines and adhesion molecules may induce inflammatory phenotypes of SMC and functionally regulate monocyte and macrophage adhesion to SMC during atherosclerosis $(2,3)$. It was found that WGE suppressed SMC proliferation and migration promoted by neighboring macrophages, likely resulting in inhibition of intimal hyperplasia of SMC. Macrophages release inflammatory cytokines and growth factors in the intimal interstitial compartments $(24,25)$. There is evidence that SMC growth is regulated by a variety of factors including angiotensin II, basic fibroblast growth factor, PDGF, and TGF- $\beta 1$ (26-29). TGF- $\beta 1$ is released during acute phases of vascular injury by degranulating platelets and is secreted during chronic phases of lesion development by SMC and activated macrophages (29-31). This study showed that PDGF may be one of the mitogenic factors being released from macrophages and enhancing SMC proliferation. In addition, TGF- $\beta 1$ released from macrophages was assumed to promote SMC proliferation. Therefore, PDGF and TGF- $\beta 1$ appeared to be involved in the intimal hyperplasia by an intimate contact of SMC with newly differentiated macrophages. WGE was effective in retarding intimal hyperplasia through suppressing release of these growth factors.

TGF- $\beta$ regulates matrix synthesis during the atherosclerotic process as a major fibrogenic factor in tissue damage (4). CTGF is a secreted matricellular protein and matrix component, and 
is thought to mediate the downstream profibrotic actions of TGF- $\beta(5,6)$. CTGF is barely expressed in normal tissues, but is strongly upregulated in fibrotic tissues, suggesting that CTGF expression is tightly regulated. However, its precise role in the interstitial fibrogenesis of vessels still needs to be clarified. In addition, CTGF is induced by other fibrogenic stimuli such as thrombin or mechanical stretch (7). CTGF induces accumulation of ECM by inducing collagen (8). In the present study, TGF- $\beta 1$ originated from macrophages activated SMC production of CTGF and collagen IV, which effects were decreased by WGE. This study showed that TGF- $\beta 1$ and macrophage-conditioned media induced TGF- $\beta$ receptors of R1 and R2 of SMC, activating its downstream effector CTGF. Accordingly, newly differentiated macrophages activated SMC to release CTGF leading to production of collagen IV in the intimal interstitial compartment. WGE inhibited SMC activation by decreasing TGF- $\beta 1$ secretion from macrophages.

The ECM degradation as well as SMC growth is a causing factor for atherosclerosis (32). MMP enzymes not only remodel ECM but also modulate cellular mediator secretion (9). The accumulation of macrophage-derived foam cells in vulnerable plaques correlates with increased MMP secretion and weak fibrous cap tissues. MMPs play important roles in the destabilization of atherosclerotic plaques (12). This study showed that macrophages caused SMC to produce MMP-2, an effect hampered by WGE without a significant impact on MT-1 MMP expression. Thus, WGE appeared to disturb plaque rupture within atherosclerotic lesions. MMP have been established to regulate SMC migration into the intimal layer of blood vessels $(13,33)$. Accordingly, MMPs have been clearly implicated in important targets of athrosclerosis therapy and SMC migration. In fact, WGE inhibited transmigration of SMC stimulated by culturing in monocyte-differentiated macrophage-conditioned media.

In summary, the present study showed that WGE suppressed SMC proliferation and migration promoted by newly differentiated macrophages within atherosclerotic plaques. WGE diminished TGF- $\beta 1$ level of macrophages and suppressed the TGF- $\beta 1$ effects on SMC exposed to macrophage-conditioned media. It is interred that the WGE inhibition of SMC proliferation and migration was mediated by dampening mitogenic and fibrogenic TGF- $\beta 1$ signaling from neighboring macrophages. In addition, WGE inhibited plaque rupture by inhibiting SMC migration and MMP-2 secretion. The ECM accumulation and intimal thickening were blunted by WGE through disturbing CTGF production of macrophages resulting in collagen IV secretion. TGF- $\beta 1$ originated from macrophages played a role in the ECM production of neighboring SMC. Taken together, anti-diabetic WGE may prevent atherosclerosis involving SMC proliferation, migration and fibrogenic activation within plaque lesions. Furthermore, these results may provide clues for developing useful therapeutic agents against vascular inflammation- and diabetes-associated pathologies.

\section{Acknowledgements}

This study was supported by the Ministry of Knowledge Economy through the Technological Development Project for the Regional Strategic Planning and by the Hallym University, Korea.

\section{References}

1. Owens GK, Kumar MS and Wamhoff BR: Molecular regulation of vascular smooth muscle cell differentiation in development and disease. Physiol Rev 84: 767-801, 2004.

2. Orr AW, Hastings NE, Blackman BR and Wamhoff BR: Complex regulation and function of the inflammatory smooth muscle cell phenotype in atherosclerosis. J Vasc Res 47: 168-180, 2010.

3. Raines EW and Ferri N: Thematic review series: the immune system and atherogenesis. Cytokines affecting endothelial and smooth muscle cells in vascular disease. J Lipid Res 46: 1081-1092, 2005.

4. Toma I and McCaffrey TA: Transforming growth factor- $\beta$ and atherosclerosis: interwoven atherogenic and atheroprotective aspects. Cell Tissue Res 347: 155-175, 2012.

5. Cicha I and Goppelt-Struebe M: Connective tissue growth factor: context-dependent functions and mechanisms of regulation. Biofactors 35: 200-208, 2009.

6. Okada H, Kikuta T, Kobayashi T, Inoue T, Kanno Y, Takigawa M, Sugaya T, Kopp JB and Suzuki H: Connective tissue growth factor expressed in tubular epithelium plays a pivotal role in renal fibrogenesis. J Am Soc Nephrol 16: 133-143, 2005.

7. Hauer-Jensen M, Wang J and Denham JW: Bowel injury: current and evolving management strategies. Semin Radiat Oncol 13: 357-371, 2003.

8. Blom IE, Goldschmeding R and Leask A: Gene regulation of connective tissue growth factor: new targets for antifibrotic therapy? Matrix Biol 21: 473-482, 2002.

9. Egeblad M and Werb Z: New functions for the matrix metalloproteinases in cancer progression. Nat Rev Cancer 2: 161-174, 2002.

10. Itoh Y and Seiki M: MT1-MMP: a potent modifier of pericellular microenvironment. J Cell Physiol 206: 1-8, 2006.

11. Sato $\mathrm{H}$ and Takino T: Coordinate action of membrane-type matrix metalloproteinase-1 (MT1-MMP) and MMP-2 enhances pericellular proteolysis and invasion. Cancer Sci 101: 843-847, 2010.

12. Rouis M: Matrix metalloproteinases: a potential therapeutic target in atherosclerosis. Curr Drug Targets Cardiovasc Haematol Disord 5: 541-548, 2005.

13. Ding Q, Chai H, Mahmood N, Tsao J, Mochly-Rosen D and Zhou W: Matrix metalloproteinases modulated by protein kinase $\mathrm{C} \varepsilon$ mediate resistin-induced migration of human coronary artery smooth muscle cells. J Vasc Surg 53: 1044-1051, 2011.

14. Leifert WR and Abeywardena MY: Cardioprotective actions of grape polyphenols. Nutr Res 28: 729-737, 2008.

15. Bertelli AA and Das DK: Grapes, wines, resveratrol, and heart health. J Cardiovasc Pharmacol 54: 468-476, 2009.

16. Dohadwala MM and Vita JA: Grapes and cardiovascular disease. J Nutr 139: S1788-S1793, 2009.

17. Choi YJ, Jeong YJ, Lee YJ, Kwon HM and Kang YH: (-) Epigallocatechin gallate and quercetin enhance survival signaling in response to oxidant-induced human endothelial apoptosis. J Nutr 135: 707-713, 2005.

18. Sprague AH and Khalil RA: Inflammatory cytokines in vascular dysfunction and vascular disease. Biochem Pharmacol 78: 539-552, 2009.

19. Hui DY: Intimal hyperplasia in murine models. Curr Drug Targets 9: 251-260, 2008

20. Fukumoto Y, Libby P, Rabkin E, Hill CC, Enomoto M, Hirouchi Y, Shiomi M and Aikawa M: Statins alter smooth muscle cell accumulation and collagen content in established atheroma of Watanabe heritable hyperlipidemic rabbits. Circulation 103: 993-999, 2001.

21. Leeuwis JW, Nguyen TQ, Theunissen MG, Peeters W, Goldschmeding R, Pasterkamp G and Vink A: Connective tissue growth factor is associated with a stable atherosclerotic plaque phenotype and is involved in plaque stabilization after stroke. Stroke 41: 2979-2981, 2010.

22. Bochaton-Piallat ML and Gabbiani G: Modulation of smooth muscle cell proliferation and migration: role of smooth muscle cell heterogeneity. Handb Exp Pharmacol 170: 645-663, 2005.

23. Bujo $\mathrm{H}$ and Saito Y: Modulation of smooth muscle cell migration by members of the low-density lipoprotein receptor family. Arterioscler Thromb Vasc Biol 26: 1246-1252, 2006.

24. Ley K, Miller YI and Hedrick CC: Monocyte and macrophage dynamics during atherogenesis. Arterioscler Thromb Vasc Biol 31: 1506-1516, 2011.

25. Badimon L, Storey RF and Vilahur G: Update on lipids, inflammation and atherothrombosis. Thromb Haemost 105 (Suppl 1): S34-S42, 2011. 
26. Daemen MJ, Lombardi DM, Bosman FT and Schwartz SM: Angiotensin II induces smooth muscle cell proliferation in the normal and injured rat arterial wall. Circ Res 68: 450-456, 1991.

27. Lindner V, Lappi DA, Baird A, Majack RA and Reidy MA: Role of basic fibroblast growth factor in vascular lesion formation. Circ Res 68: 106-113, 1991.

28. Wada Y, Kato S, Okamoto K, Izumaru S, Aoyagi S and Morimatsu M: Diltiazem, a calcium antagonist, inhibits matrix metalloproteinase-1 (tissue collagenase) production and collagenolytic activity in human vascular smooth muscle cells. Int J Mol Med 8: 561-566, 2001.

29. Ikedo H, Tamaki K, Ueda S, Kato S, Fujii M, Ten Dijke P and Okuda S: Smad protein and TGF- $\beta$ signaling in vascular smooth muscle cells. Int J Mol Med 11: 645-650, 2003.
30. Assoian R and Sporn MB: Type beta transforming growth factor in human platelets: release during platelet degranulation and action on vascular smooth muscle cells. J Cell Biol 102: 1217-1223, 1986.

31. Assoian RK, Fleurdelys BE, Stevenson HC, Miller PJ, Madtes DK, Raines EW, Ross R and Sporn MB: Expression and secretion of type beta transforming growth factor by activated human macrophages. Proc Natl Acad Sci USA 84: 6020-6024, 1987.

32. Ross R: The pathogenesis of atherosclerosis: a perspective for the 1990s. Nature 362: 801-809, 1993.

33. Newby AC and Zaltsman AB: Molecular mechanisms in intimal hyperplasia. J Pathol 190: 300-309, 2000. 